\title{
Systematic elucidation of the mechanism of geraniol via network pharmacology
}

This article was published in the following Dove Medical Press journal:

Drug Design, Development and Therapy

\section{Yun-Fei Zhang* \\ Yue Huang* \\ Yi-Hua $\mathrm{Ni}$ \\ Zheng-Min Xu}

Department of Otolaryngology-Head and Neck Surgery, Children's Hospital of Fudan University, Shanghai, People's Republic of China

*These authors contributed equally to this work
Correspondence: Zheng-Min Xu Department of Otolaryngology-Head and Neck Surgery, Children's Hospital of Fudan University, 399 Wan Yuan Road, Shanghai 201 I02, People's Republic of China

Email xuzhengminsh@126.com
Background: Geraniol is an acyclic monoterpene alcohol, which is extracted from the ethereal oils of aromatic plants. A systematic analysis of its mechanism of action has not yet been carried out.

Methods: In this study, the druggability of geraniol was assessed via Traditional Chinese Medicine Systems Pharmacology Database (TCMSP), and the potential targets of geraniol were identified using the Comparative Toxicogenomics Database (CTD). Additionally, Gene Ontology (GO) and Kyoto Encyclopedia of Genes and Genomes (KEGG) pathway analyses were performed using WebGestalt. Drug-target-pathway networks were constructed using Cytoscape to give a visual view.

Results: Our findings showed that geraniol has superb druggability with 38 putative identified target genes. GO, KEGG, and network analyses revealed that these targets were associated with cancer, inflammatory immunoreactions, and other physiological processes.

Conclusion: Geraniol is predicted to target multiple proteins and pathways that shape a network which can exert systematic pharmacological effects.

Keywords: geraniol, druggability, target prediction, enrichment analysis, network pharmacology

\section{Introduction}

Natural products and traditional Chinese medicine (TCM) are the most abundant resources of active compounds for drug discovery. Monoterpenes, for example, are dietary compounds extracted from the ethereal oils of many vegetables, fruits, and especially TCM. Geraniol (Figure 1A) is an acyclic monoterpene alcohol, which is found in the ethereal oils of aromatic plants. ${ }^{1}$ Geraniol has been shown to exert a wide spectrum of pharmacological activities, for example anti-inflammatory, antimicrobial, antitumor, and so on. ${ }^{2-5}$ Close attention has been paid to geraniol due to its potential role in the treatment of a variety of diseases, such as chronic or allergic rhinitis, lung cancer, etc. ${ }^{6-8}$ These results suggest that geraniol can be utilized as a valuable chemical probe or a chemical moiety for the dissection of complex biological processes, discovery of hidden molecular relationships, and identification of therapeutic target molecules and pathways. Accordingly, the molecular mechanisms which geraniol induces and the resulting changes in cellular phenotypes are rarely studied. Meanwhile, employment of computational methodologies for the identification of drug targets and the underlying mechanisms is becoming mainstream in order to save money, time, and effort. ${ }^{9,10}$ In particular, computational target identification and following the molecular mechanisms can accelerate the drug discovery and drug design processes.

Therefore, we elucidated the pharmacological actions of geraniol systematically using computational methodologies. First, the druggability of geraniol was assessed 
A

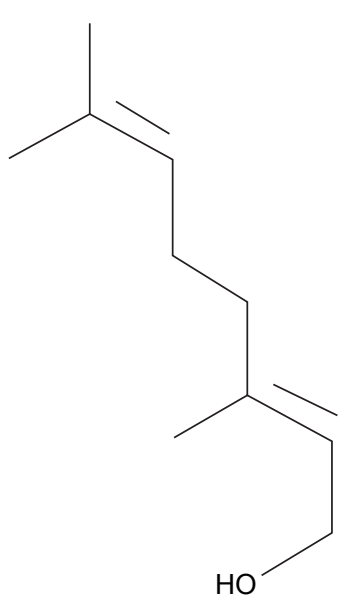

B

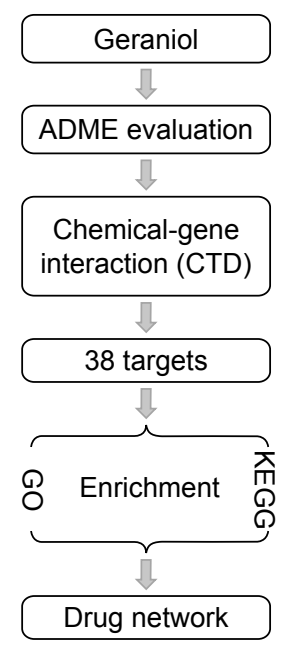

Figure I (A) Chemical structure of geraniol downloaded from the PubChem database (CID: 637566); (B) Workflow for the identification of potential geraniol target genes that integrates ADME evaluation, chemical-gene interaction, GO and KEGG pathway analyses and network construction.

Abbreviations: ADME, absorption, distribution, metabolism, and excretion; GO, gene ontology; KEGG, Kyoto Encyclopedia of Genes and Genomes; CTD, Comparative Toxicogenomics Database.

using the Traditional Chinese Medicine Systems Pharmacology Database (TCMSP) server. ${ }^{11}$ Next the potential candidate target genes were predicted by chemical-gene interaction analysis. ${ }^{12}$ Furthermore, gene ontology and pathway analyses were investigated using the identified target genes. Finally, drug-target network was constructed to provide a systematic overview of the potential target genes and the mechanism of action for geraniol. A schematic diagram of the analysis procedures for geraniol target gene prediction is shown in Figure 1B.

\section{Materials and methods}

Assessment of pharmacokinetics properties using TCMSP database (http://sp.nwu.edu.cn/tcmsp.php) is a resource of systems pharmacology for TCMs or related compounds. ${ }^{11}$ It can provide information on the absorption, distribution, metabolism, and excretion (ADME) properties of a drug with potential biological effects at a systematic level, for example, oral bioavailability (OB), drug likeness (DL), Caco-2 permeability (Caco-2), blood-brain barrier (BBB), and so on. ${ }^{13,14}$

Of all pharmacokinetics properties, $\mathrm{OB}$ is the foremost feature of orally administered drugs, since it acts as a vital role in evaluating the efficacy of the drug distribution to the systemic circulation. In TCMSP database, OB was calculated on OBioavaill.1 based on an in-house model. ${ }^{11,13}$ For orally administered drugs, the movement across the intestinal epithelial barrier is one of the biggest obstacles of human absorption and its bioavailability. ${ }^{11,13}$ In present study, the chemical name "geraniol" was entered to the search box
Table I Putative targets of geraniol

\begin{tabular}{|c|c|c|c|}
\hline Num. & $\begin{array}{l}\text { Gene } \\
\text { ID }\end{array}$ & $\begin{array}{l}\text { Gene } \\
\text { symbol }\end{array}$ & Gene name \\
\hline $\mathrm{I}$ & 1260 & CNGA2 & Cyclic nucleotide-gated channel alpha 2 \\
\hline 2 & 1350 & COX7C & Cytochrome $\mathrm{c}$ oxidase subunit 7C \\
\hline 3 & 1537 & $\mathrm{CYCl}$ & Cytochrome cl \\
\hline 4 & 1555 & CYP2B6 & $\begin{array}{l}\text { Cytochrome P450 family } 2 \text { subfamily B } \\
\text { member } 6\end{array}$ \\
\hline 5 & 2099 & ESRI & Estrogen receptor I \\
\hline 6 & 2100 & ESR2 & Estrogen receptor 2 \\
\hline 7 & 2554 & GABRAI & $\begin{array}{l}\text { Gamma-aminobutyric acid type } \mathrm{A} \\
\text { receptor alphal subunit }\end{array}$ \\
\hline 8 & 2560 & GABRB I & $\begin{array}{l}\text { Gamma-aminobutyric acid type } A \\
\text { receptor betal subunit }\end{array}$ \\
\hline 9 & 2641 & GCG & Glucagon \\
\hline 10 & 26762 & HAVCRI & Hepatitis A virus cellular receptor I \\
\hline II & 2769 & GNA I5 & G protein subunit alpha 15 \\
\hline 12 & 2774 & GNAL & G protein subunit alpha $L$ \\
\hline 13 & 2936 & GSR & Glutathione-disulfide reductase \\
\hline 14 & 3162 & HMOXI & Heme oxygenase I \\
\hline 15 & 3265 & HRAS & HRas proto-oncogene, GTPase \\
\hline 16 & 3575 & IL7R & Interleukin 7 receptor \\
\hline 17 & 3576 & CXCL8 & C-X-C motif chemokine ligand 8 \\
\hline 18 & 3949 & $L D L R$ & Low density lipoprotein receptor \\
\hline 19 & 43 & ACHE & Acetylcholinesterase \\
\hline 20 & 4790 & NFKB I & Nuclear factor kappa B subunit I \\
\hline 21 & 4953 & $O D C I$ & Ornithine decarboxylase I \\
\hline 22 & 5111 & PCNA & Proliferating cell nuclear antigen \\
\hline 23 & 5594 & MAPKI & Mitogen-activated protein kinase I \\
\hline 24 & 5595 & MAPK2 & Mitogen-activated protein kinase 2 \\
\hline 25 & 5743 & PTGS2 & Prostaglandin-endoperoxide synthase 2 \\
\hline 26 & 581 & $B A X$ & $\mathrm{BCL} 2$ associated $\mathrm{X}$, apoptosis regulator \\
\hline 27 & 5894 & RAFI & $\begin{array}{l}\text { Raf-I proto-oncogene, serine/threonine } \\
\text { kinase }\end{array}$ \\
\hline 28 & 596 & $B C L 2$ & $\mathrm{BCL2}$, apoptosis regulator \\
\hline 29 & 7083 & TKI & Thymidine kinase I \\
\hline 30 & 7157 & TP53 & Tumor protein $p 53$ \\
\hline 31 & 7298 & TYMS & Thymidylate synthetase \\
\hline 32 & 7442 & TRPVI & $\begin{array}{l}\text { Transient receptor potential cation } \\
\text { channel subfamily } \mathrm{V} \text { member }\end{array}$ \\
\hline 33 & 836 & CASP3 & Caspase 3 \\
\hline 34 & 8383 & ORIAI & $\begin{array}{l}\text { Olfactory receptor family I subfamily A } \\
\text { member I }\end{array}$ \\
\hline 35 & 8390 & ORIGI & $\begin{array}{l}\text { Olfactory receptor family I subfamily G } \\
\text { member I }\end{array}$ \\
\hline 36 & 841 & CASP8 & Caspase 8 \\
\hline 37 & 842 & CASPQ & Caspase 9 \\
\hline 38 & 847 & CAT & Catalase \\
\hline
\end{tabular}

and its pharmacokinetic properties were investigated at the molecular level.

\section{Target identification by the Comparative Toxicogenomics Database}

The Comparative Toxicogenomics Database (CTD, http:// ctdbase.org/), is a robust, publicly available database for toxicogenomic information. It provides manually curated key information about chemical-gene/protein interactions, 
chemical-disease and gene-disease relationships, from peer-reviewed scientific literature. Currently, CTD includes more than 30.5 million toxicogenomic relationships related to chemicals, proteins, and so on. ${ }^{12}$ Given a compound, CTD can provide the corresponding target genes sorted by the interactions between them by descending order. Candidate targets of geraniol were predicted using CTD with default parameters.

\section{Analysis by GeneMANIA}

GeneMANIA (http://www.genemania.org), is an userfriendly and flexible web server, for generating hypotheses in regard to gene function, analyzing gene lists, and prioritizing genes for functional assays. ${ }^{15}$

Given a query list, GeneMANIA can list the genes that have shared properties, or function similarly with the original query. It also shows a functional relationship network, expounding the association among the list as well as curated genomics and proteomics data. The potential candidate target genes were entered into the search bar after selecting Homo sapiens from the organism option, and the results were further collated.

\section{Gene function and pathway enrichment analysis}

Web-based gene set analysis toolkit (WebGestalt, http:// www.webgestalt.org/option.php) can be utilized to thoroughly understand the functional and pathway enrichment information on the gene of interest. ${ }^{16}$ Potential candidate targets were input to the WebGestalt server using overrepresentation enrichment analysis method with the Gene Ontology (GO) and Kyoto Encyclopedia of Genes and Genomes (KEGG) databases.

GO analysis is a commonly used approach for annotating genes and gene products with functions including molecular function, biological pathways, and cellular components. ${ }^{17}$ KEGG is a useful resource for systematic analysis of gene functions and related high-level genome functional information. ${ }^{18,19}$

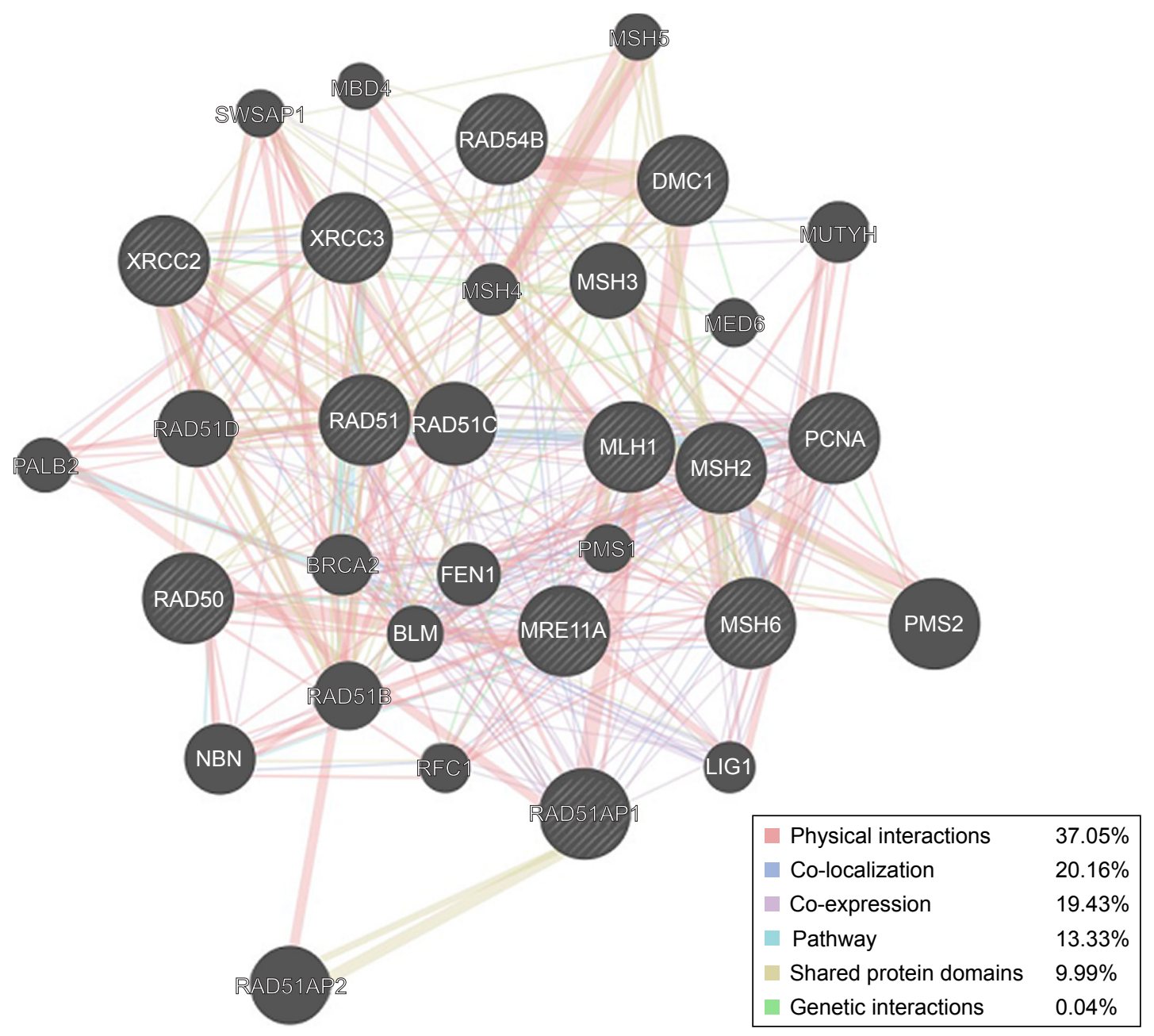

Figure 2 Protein network of geraniol. Black nodes represent target proteins, and connecting colors indicate different correlations. Functional associations between targets were investigated using GeneMANIA. Genes in black circles were query terms while these in gray circle indicate genes associated with query genes. 

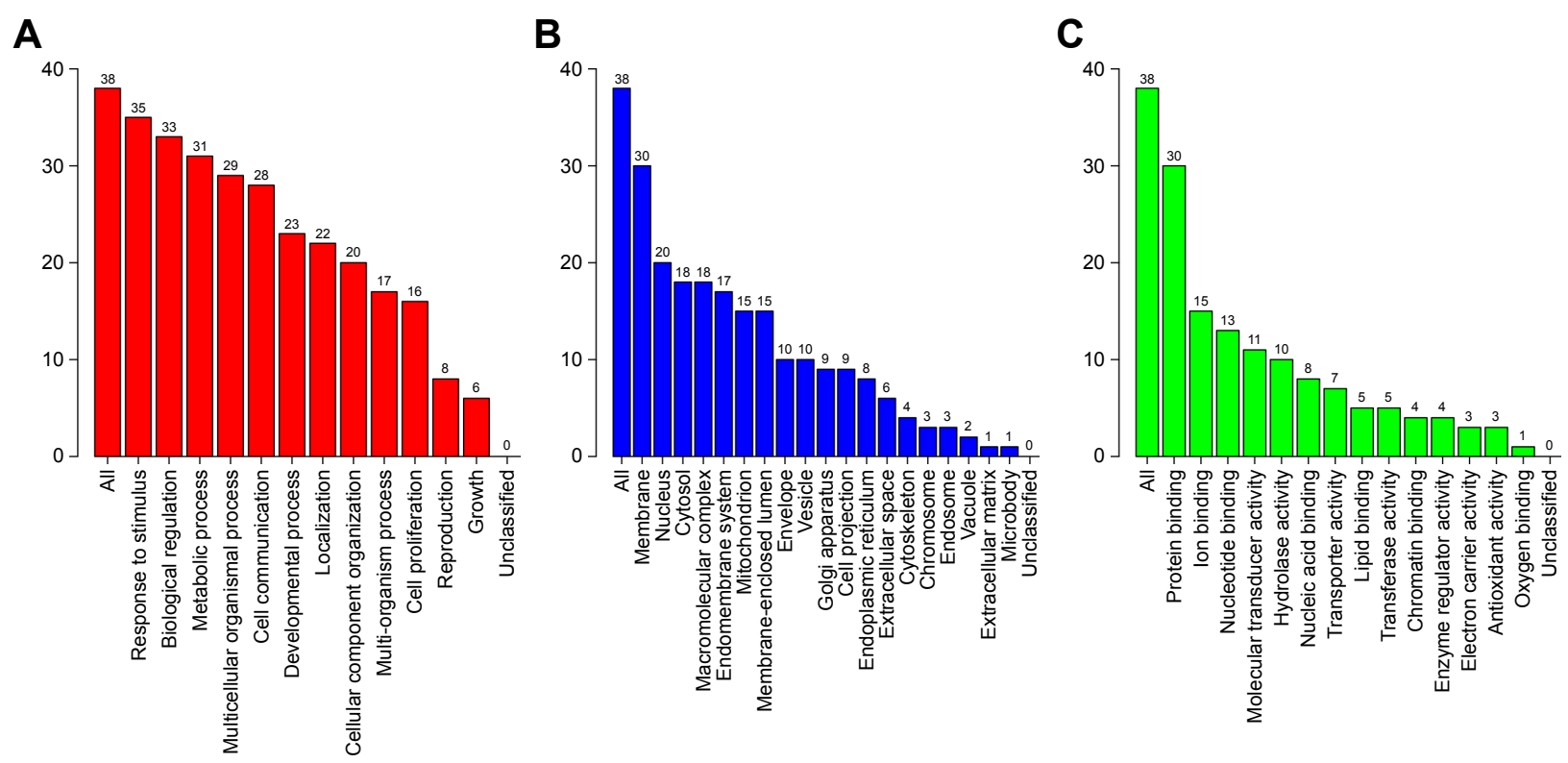

Figure $3 \mathrm{GO}$ map of putative target genes. (A) Biological process categories. (B) Cellular component categories. (C) Molecular function categories.

\section{Network construction}

In order to understand the complex relationships among compound, targets, and diseases, we used Cytoscape (v 3.6.1; https://www.nigms.nih.gov/) to construct and analyze the three-layer networks.

\section{Results}

Pharmacokinetics properties of geraniol

ADME describes the disposition of a pharmaceutical compound and TCMCP provides information on 12 very important characteristics on ADME-related properties like

hsa04933-AGE-RAGE signaling pathway in diabetic complications

hsa05200-pathways in cancer

hsa05219-bladder cancer

hsa04917-prolactin signaling pathway

hsa05161-hepatitis B

hsa04210-apoptosis

hsa05210-colorectal cancer

hsa01522-endocrine resistance

hsa01524-platinum drug resistance

hsa05215-prostate cancer

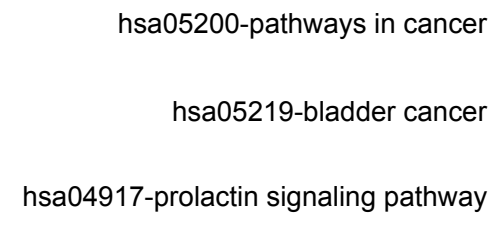

0

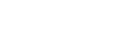

2

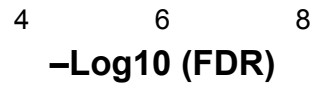

10

Figure 4 KEGG pathway analysis of putative target genes. 


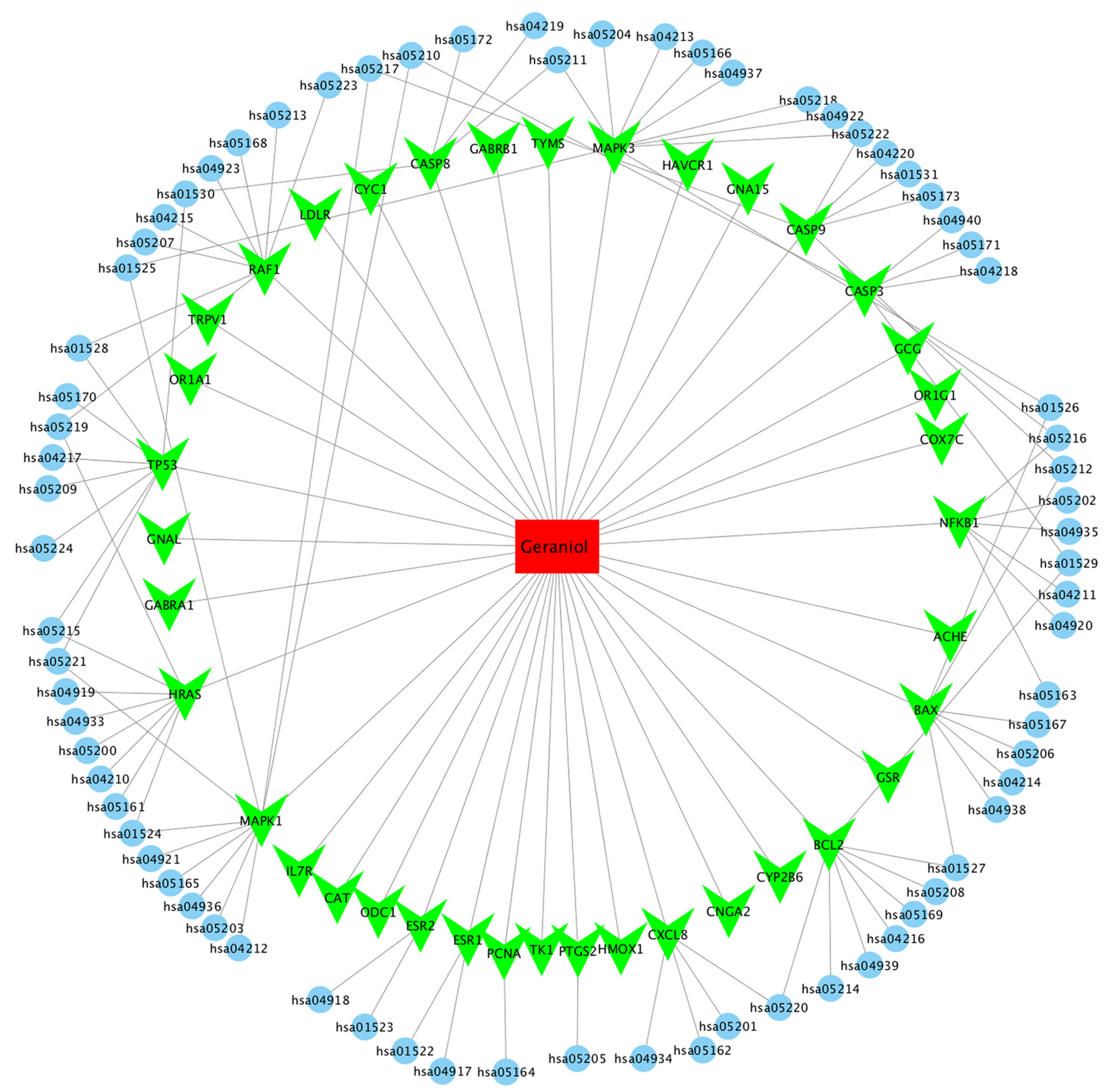

Figure 5 Geraniol-target-pathway network.

Caco-2, human OB, BBB, and Lipinski's rule of five for drug screening and evaluation. ${ }^{11}$ AMDE-related properties of geraniol were thoroughly investigated using TCMSP. Notably, the OB of geraniol was calculated to be $23.93 \%$.

\section{Targets identification of geraniol}

Potential targets of geraniol were predicted using the CTD as described in the "Materials and methods" section. ${ }^{12}$ In total, 41 candidate target genes were identified by CTD. Afterwards, we filtered these genes by using the threshold chemical-gene interaction $\geq 1$ and removed the non-human genes. Finally, 38 unique target genes for geraniol remained (Table 1). These 38 identified interacting genes were used for further investigation.

\section{GeneMANIA analysis}

Among the 38 targets and their interacting proteins, it was found that $37.05 \%$ had physical interactions, $20.16 \%$ exerted co-localization, and $19.43 \%$ displayed similar coexpression characteristics. Other results, including pathway, 
Table 2 Pharmacological and molecular properties of geraniol

\begin{tabular}{l|l|l|l|l|l|l|l|l|l|l|l}
\hline Name & MW & AlogP & Hdom & Hacc & OB (\%) & Caco-2 & BBB & DL & FSAF & TPSA & RBN \\
\hline Geraniol & 154.28 & 2.93 & $\mathrm{I}$ & $\mathrm{I}$ & 23.93 & 1.19 & 1.14 & 0.02 & 0.27 & 20.23 & 4 \\
\hline
\end{tabular}

Abbreviations: Caco-2, Caco-2 permeability; OB, oral bioavailability; DL, drug likeness; BBB, blood-brain barrier.

shared protein domains and genetic interactions, are shown in Figure 2.

\section{GO and pathway analysis}

In order to study further the 38 identified target genes, GO and KEGG enrichment analyses were carried out using WebGestalt. As shown in Figure 3, the top seven functions were used as response to stimulus (35/38), biological regulation (33/38), metabolic process (31/38), membrane (30/38), protein binding (30/38), multicellular organismal process (29/38), and cell communication (28/38). These functional terms are highly relevant to anti-inflammatory activities, especially for chronic or allergic rhinitis.

As for pathway analysis, the 38 targets participate in 10 KEGG pathways with significant false discovery rate (FDR)-adjusted $P$-value including apoptosis, pathways in cancer, and so on, which were shown in Figure 4.

\section{Network analysis}

Based on target and pathway analyses, an entire compound, targets and diseases network was constructed using Cytoscape (v 3.6.1). As shown in Figure 5, this compound, targets, and diseases interaction network has 80 nodes and 129 edges. The red oblong, green inverted triangles, and blue circles correspond to geraniol, target genes, and pathways, respectively.

\section{Discussion}

Poor pharmacokinetics and toxicity are the most important causes of costly delays in drug discovery and development. There is, therefore, growing belief that certain features in the drug discovery process should be prioritized. ${ }^{20}$ In silico analysis can improve predictions and pharmacokinetic modeling, as well as metabolic and toxicity endpoints; all of which accelerate and streamline the drug discovery process. ${ }^{9,10}$

Lipinski's rule of five can identify some very important drug properties, which should be taken into account for compounds developed with the aim of oral delivery. ${ }^{21}$ The rule of five takes into consideration molecular weights (MWs) $<500 \mathrm{Da}$, a $\log \mathrm{P}<5$, as well as numbers of hydrogen-bond donors and acceptors less than 5 and 10 , respectively. Today, the rule of five is generally referred to as a guideline for drug optimization. ${ }^{22}$ As shown in Table 2, the pharmacokinetic properties of geraniol meet these requirements, meaning geraniol is a superior candidate for drug development.

In drug discovery, target gene identification is the first step. More and more active compounds or drugs are being shown to interact with multiple genes or proteins. ${ }^{23-26}$ A variety of in silico target identification approaches have been developed and are broadly applied toward this aim. As listed in Table 1, 38 potential targets of geraniol were identified using computational methods. The results of GeneMANIA provided information on physical interactions, co-localization, co-expression as well as shared protein domains, and implied that the targets and their interacting proteins may have identical or similar functions.

We identified an inflammatory role for geraniol in allergic rhinitis. Similarly, Madankumar et al reported geraniol exerts antimicrobial, antioxidant, antitumor, and anti-inflammatory activities via activation of apoptotic pathways. ${ }^{6,27}$ These results closely coincide with our findings from $\mathrm{GO}$ and KEGG analyses.

The drug-target network shown in Figure 5 also revealed that geraniol has multiple targets and further indicated that it possesses multiple pharmacological activities. Cho et al have also revealed that geraniol exerts systematic pharmacological effects by targeting multiple proteins and pathways..$^{28,29}$ Multiple target therapeutic medicaments are more effective for the treatment of complex diseases, for instance allergic rhinitis, cancers, and are less vulnerable to adaptive resistance. Hence, geraniol could be a promising resource that may be utilized as chemical moiety, lead compound, or an active ingredient for future drug discovery.

In summary, we would like to underline that geraniol is an active ingredient or a promising compound for the development of a safe and effective multi-targeted anticancer medicament. This study provides novel insight into the perspectives and challenges for geraniol research and its application in future clinical investigation.

\section{Disclosure}

The authors report no conflicts of interest in this work. 


\section{References}

1. Lapczynski A, Bhatia SP, Foxenberg RJ, Letizia CS, Api AM. Fragrance material review on geraniol. Food Chem Toxicol. 2008;46(Suppl 11): S160-S170. doi:10.1016/j.fct.2008.06.048

2. Solórzano-Santos F, Miranda-Novales MG. Essential oils from aromatic herbs as antimicrobial agents. Curr Opin Biotechnol. 2012;23(2): 136-141. doi:10.1016/j.copbio.2011.08.005

3. Tiwari M, Kakkar P. Plant derived antioxidants-Geraniol and camphene protect rat alveolar macrophages against t-BHP induced oxidative stress. Toxicol In vitro. 2009;23(2):295-301. doi:10.1016/j.tiv.2008.12.014

4. de Carvalho KI, Bonamin F, Dos Santos RC, et al. Geraniol-a flavoring agent with multifunctional effects in protecting the gastric and duodenal mucosa. Naunyn Schmiedebergs Arch Pharmacol. 2013;387(4): 355-365. doi:10.1007/s00210-013-0947-z

5. Rekha KR, Selvakumar GP, Sethupathy S. Geraniol ameliorates the motor behavior and neurotrophic factors inadequacy in MPTP-induced mice model of Parkinson's disease. J Mol Neurosci. 2013;51(3): 851-862. doi:10.1007/s12031-013-0074-9

6. Huang Y, Yang XL, Ni YH, Xu ZM. Geraniol suppresses proinflammatory mediators in phorbol 12-myristate 13-acetate with A23187-induced HMC-1 cells. Drug Des Dev Ther. 2018;12:2897-2903. doi:10.2147/ DDDT.S145702

7. Ong TP, Heidor R, de Conti A, Dagli MLZ, Moreno FS. Farnesol and geraniol chemopreventive activities during the initial phases of hepatocarcinogenesis involve similar actions on cell proliferation and DNA damage, but distinct actions on apoptosis, plasma cholesterol and HMGCoA reductase. Carcinogenesis. 2006;27(6):1194-1203. doi:10. 1093/carcin/bgi291

8. Galle M, Crespo R, Kladniew BR, Villegas SM, Polo M, de Bravo MG. Suppression by geraniol of the growth of A549 human lung adenocarcinoma cells and inhibition of the mevalonate pathway in culture and in vivo: potential use in cancer chemotherapy. Nutr Cancer. 2014; 66(5):888-895. doi:10.1080/01635581.2014.916320

9. Bi YH, Zhang LH, Chen SJ, Page GL. Antitumor mechanisms of curcumae rhizoma based on network pharmacology. Evid Based Compl Altern Med. 2018;2018:9. doi:10.1155/2018/9567061

10. Lin YC, Chang CW, Wu CR. Analysis of the action mechanism of Fang Ji Huang Qi decoction in treating rheumatoid arthritis by network pharmacology. Modernization Traditional Med. 2018;3(6):286-294.

11. Ru J, Li P, Wang J, et al. TCMSP: a database of systems pharmacology for drug discovery from herbal medicines. J Cheminform. 2014;6:13. doi:10.1186/1758-2946-6-6

12. Davis AP, Grondin CJ, Johnson RJ, et al. The comparative toxicogenomics database: update 2017. Nucleic Acids Res. 2017;45(D1):D972-D978. doi:10.1093/nar/gkw838

13. Pei T, Zheng C, Huang C, et al. Systematic understanding the mechanisms of vitiligo pathogenesis and its treatment by Qubaibabuqi formula. J Ethnopharmacol. 2016;190:272-287. doi:10.1016/j.jep.2016. 06.001

14. Zhang W, Tao Q, Guo Z, et al. Systems pharmacology dissection of the integrated treatment for cardiovascular and gastrointestinal disorders by traditional Chinese medicine. Sci Rep. 2016;6:32400. doi:10.1038/ srep32400
15. Warde-Farley D, Donaldson SL, Comes O, et al. The GeneMANIA prediction server: biological network integration for gene prioritization and predicting gene function. Nucleic Acids Res. 2010;38(Web Server issue):W214-W220. doi:10.1093/nar/gkq537

16. Wang J, Duncan D, Shi Z, Zhang B. WEB-based GEne SeT AnaLysis Toolkit (WebGestalt): update 2013. Nucleic Acids Res. 2013;41(Web Server issue):W77-W83. doi:10.1093/nar/gkt439

17. The Gene Ontology Consortium. Expansion of the gene ontology knowledgebase and resources. Nucleic Acids Res. 2017;45(D1): D331-D338. doi:10.1093/nar/gkw1108

18. Kanehisa M, Furumichi M, Tanabe M, Sato Y, Morishima K. KEGG: new perspectives on genomes, pathways, diseases and drugs. Nucleic Acids Res. 2017;45(D1):D353-D361. doi:10.1093/nar/gkw1092

19. Kanehisa M, Goto S. KEGG: Kyoto encyclopedia of genes and genomes. Nucleic Acids Res. 2000;28(1):27-30.

20. van de Waterbeemd H, Gifford E. ADMET in silico modelling: towards prediction paradise? Nat Rev Drug Discov. 2003;2(3):192-204. doi: $10.1038 /$ nrd1032

21. Lipinski CA, Lombardo F, Dominy BW, Feeney PJ. Experimental and computational approaches to estimate solubility and permeability in drug discovery and development settings. Adv Drug Deliv Rev. 2001; 46(1-3):3-26.

22. Leeson PD. Molecular inflation, attrition and the rule of five. Adv Drug Deliv Rev. 2016;101:22-33. doi:10.1016/j.addr.2016.01.018

23. Schenone M, Dančík V, Wagner BK, Clemons PA. Target identification and mechanism of action in chemical biology and drug discovery. Nat Chem Biol. 2013;9(4):232-240. doi:10.1038/nchembio.1199

24. Wang L, Xie XQ. Computational target fishing: what should chemogenomics researchers expect for the future of in silico drug design and discovery? Future Med Chem. 2014;6(3):247-249. doi:10.4155/ fmc. 14.5

25. Hopkins AL. Network pharmacology: the next paradigm in drug discovery. Nat Chem Biol. 2008;4(11):682-690. doi:10.1038/nchembio.118

26. Wang J, Gao L, Lee YM, et al. Target identification of natural and traditional medicines with quantitative chemical proteomics approaches. Pharmacol Ther. 2016;162:10-22. doi:10.1016/j. pharmthera.2016.01.010

27. Madankumar A, Tamilarasi S, Premkumar T, Gopikrishnan M, Nagabhishek N, Devaki T. Geraniol attenuates 4NQO-induced tongue carcinogenesis through downregulating the activation of NF-kappaB in rats. Mol Cell Biochem. 2017;434(1-2):7-15. doi:10.1007/s11010-0173030-0

28. Cho M, So I, Chun JN, Jeon JH. The antitumor effects of geraniol: modulation of cancer hallmark pathways (Review). Int J Oncol. 2016; 48(5):1772-1782. doi:10.3892/ijo.2016.3427

29. Crespo R, Montero Villegas S, Abba MC, de Bravo MG, Polo MP. Transcriptional and posttranscriptional inhibition of HMGCR and PC biosynthesis by geraniol in 2 Hep-G2 cell proliferation linked pathways. Biochem Cell Biol. 2013;91(3):131-139. doi:10.1139/bcb-2012-0076

\section{Publish your work in this journal}

Drug Design, Development and Therapy is an international, peerreviewed open-access journal that spans the spectrum of drug design and development through to clinical applications. Clinical outcomes, patient safety, and programs for the development and effective, safe, and sustained use of medicines are the features of the journal, which

\section{Dovepress}

has also been accepted for indexing on PubMed Central. The manuscript management system is completely online and includes a very quick and fair peer-review system, which is all easy to use. Visit http://www.dovepress.com/testimonials.php to read real quotes from published authors. 